\title{
Appalachỉan
}

B O O N E, N O R T H C R O L I N A

\section{Role Of Cerebellum In Deglutition And Deglutition Disorders}

\author{
Balaji Rangarathnam \& Erin Kamarunas \& Gary H. McCullough
}

\begin{abstract}
ABTRACT: The objective of this review is to gather available evidence regarding the role of the cerebellum in swallowing- related functions. We reviewed literature on cerebellar functions related to healthy swallowing, patterns of dysphagia in individuals with cerebellar lesions, and the role of the cerebellum in therapeutic intervention of neurogenic dysphagia since 1980. A collective understanding of these studies suggests that both hemispheres of the cerebellum, predominantly the left, participate in healthy swallowing. Also, it appears that the cerebellum contributes to specific physiological functions within the entire act of swallowing, but this is not clearly understood. The understanding of patterns of dysphagia in cerebellar lesions remains ambiguous with equivocal results across a small number of studies. The cerebellum appears to be involved in oral exercises for dysphagia in the relationship between oral movements in such exercises, and deglutition remains uncertain. There is increasing evidence to suggest successful use of transcranial magnetic stimulation of the cerebellum to improve neuromotor control of swallowing. Future studies should address activation of the cerebellum with swallowing of different consistencies and tastes in healthy adults to gain better insights. Studies should also investigate dynamics of neural activation during different stages of recovery from dysphagia following strokes to cortical centers to determine if the cerebellum plays a compensatory role during instances of increased neural demands.
\end{abstract}

Rangarathnam, B., Kamarunas, E., \& McCullough, G.H. (2014). "Role Of Cerebellum In Deglutition And Deglutition Disorders." Cerebellum 13(6):767-76. [ISSN: 14734222] Version of record available at www.springer.com [DOI 10.1007/s12311-014-0584-1] 


\section{$\underline{\text { Role Of Cerebellum In Deglutition And Deglutition Disorders }}$}

Swallowing is a complex process that involves voluntary and reflexive motor control, intraoral sensory processing, sensorimotor integration, salivation, and visceral regulation [1], all culminating in the ingestion of food and liquid safely and without aspiration. Swallowing is accomplished by a complex interplay of sensory and motor functions in the oral and pharyngeal cavities. After the bolus is formed in the oral cavity, it is propelled posteriorly and a series of motor movements occur sequentially in response to sensory input regarding the bolus. These pharyngeal events include the closure of the velopharynx, retraction of the base of the tongue to the posterior pharyngeal wall, elevation of the hyoid bone, closure of the laryngeal vestibule via inversion of the epiglottis and action of true and false vocal folds, stripping of the pharyngeal constrictor muscles, and opening of the upper esophageal sphincter. Functional swallowing requires contribution from many different systems and levels including several cortical and subcortical structures, the brain stem, five pairs of cranial nerves, and various muscles and bones of the face and neck. Impairment occurring at any one of these various levels could potentially cause a disruption in the normal processes of swallowing, known as dysphagia. Dysphagia can be characterized as an abnormality of bolus flow wherein bolus flow is either stopped in the oropharynx, creating oropharyngeal residue or misdirected into the airway, i.e., penetration or aspiration [2]. 
Numerous regions have been implicated in the swallowing act. These include the precentral gyrus (MI), postcentral gyrus (SI), premotor area (PMA), supplemental motor area (SMA), anterior cingulate cortex (ACC), operculum, insula, precuneus, cuneus, prefrontal area, temporal cortex, cerebellum, brain stem, frontal cortex, internal capsule, association areas, thalamus, and the basal ganglia [1, 3-13]. Research utilizing technologies such as positron emission tomography (PET), functional magnetic resonance imaging (fMRI), and transcranial magnetic stimulation (TMS) has begun to clarify the areas of involvement and the primary pathways of neurologic activity, but many of the complex interconnections and pathways supporting and regulating deglutition remain illdefined.

A role for the cerebellum in deglutition and neurologic disorders of deglutition has become increasingly evident, but with few data points from pure cerebellar lesions, the exact nature of this role remains somewhat elusive. The purpose of this paper is to highlight current understanding and emphasize where future research may prove valuable. We employed MEDLINE, PubMed, and Google Scholar to identify studies that have addressed cerebellar involvement in swallowing with the search words "swallowing," "deglutition," "deglutition disorders," and "cerebellum" from 1980 to present. The search generated a total of 134 studies. Of these results, we filtered studies in the English language that reported involvement of the cerebellum in healthy adults with normal swallowing and patterns of swallowing impairment in adults with a lesion to the cerebellum. With these criteria, 24 studies were finally included for the review. A brief review of cortical control of deglutition is presented before discussing the role of the cerebellum.

Normal Neurologic Control of Deglutition

\section{Cortical Control}

The urge to swallow begins as sensory information from the pharynx that travels up the spinal column, through the brain stem, and to the thalamus, which then projects the information to specific regions of the somatosensory areas of the parietal cortex, where the sensation of the bolus from the back of the mouth registers [14]. A majority of the studies examining cortical activation and deglutition reported activation of SI for both volitional and automatic swallowing [1, 3-5, 7-13]. The sensory strip is somatotopically mapped with regions corresponding to the face, tongue, pharyngeal, laryngeal, and esophageal areas being located caudolaterally [4, 10]. This area is responsible for the processing of oropharyngeal stimulation created by movements of the tongue, jaw, palate, and pharyngeal musculature [7]. Several studies have indicated that stimulation to the lateral portions of SI, with its many direct projections to the nucleus of the tractus solitarius (NTS) of the brainstem [10], can evoke a swallow response [4]. Several studies have indicated that the SI also plays a modulatory role in swallowing motor movements by way of connections to both MI and the insula [3, 15], which allows for monitoring and potential alteration of the intensity of muscle contractions [8]. This demonstrates the importance of sensory feedback in the regulation of both volitional and automatic swallowing [7].

A majority of the studies examining cortical activation and deglutition have also reported activation of ACC, SMA, and/ or PMA with volitional swallowing [1, 3, 4, 6, 8-12]. It has been suggested that there is parallel processing of both swallowing and tongue movement between the SMA and SI [16]. The activation of the ACC early in the swallowing sequence is not surprising as it is reported to indicate an attentional aspect of swallowing $[3,4,15]$. A majority of the deglutition mapping studies reported activation of the ACC for volitional swallowing [3-13]. The ACC plays an important role in choosing a sensorimotor plan [11], as well as coding movement initiation mode, movement type, and rate [8]. The SMA, having been activated for premotor aspects of swallowing and receiving subcortical input from the basal ganglia, in turn, activates the precentral gyrus, or MI [14]. The SMA and MI share a substantial connection. MI and the SMA also receive cortical input from SI, the posterior parietal cortex, the thalamus, and the cerebellum.

\section{Brainstem Control}

Sensory information from the pharynx is relayed by afferent fibers, specifically CN V, VII, IX, and X, which all converge and terminate in the NTS in the medulla of the brainstem [17]. As this sensory information is relayed to the NTS, it is also sent by afferent fibers to different regions of the cerebrum, and this begins the cortical response to swallowing. The nucleus ambiguus (NA) in the medulla is the principle motor nucleus that provides input to the cranial nerves, specifically CN IX, $\mathrm{X}$, and XI, that are responsible for innervating the pharyngeal muscles of swallowing. The NTS also works to synchronize motor outputs from the NA and CN X.

Within the medulla, there are also "interneurons," known as the central pattern generator (CPG), that bidirectionally communicate with afferent and efferent levels and can initiate and/or organize the swallowing motor sequence [15]. These interneurons of the CPG are located in the NTS, in the proximate reticular formation surrounding the NTS, and in the reticular formation around and above the NA [15].

\section{Additional Areas of Neural Control}

Research continues to clarify the role of the insula and opercular cortex in deglutition. The insula is located directly 
beneath the junction of the frontal, temporal, and parietal operculum, deep in the lateral sulcus [18]. A majority of neuroimaging studies have implicated the insular cortex in both volitional and automatic swallowing, and the majority has implicated insular lateralization on the right [1, 4-7, 9-13]. The insula is reported to share connections with many cortical and subcortical structures including SI, S2, PMA, SMA, basal ganglia, thalamus, cingulate, NTS, operculum, and gustatory, olfactory, limbic, and autonomic structures [7, $11,18]$ and is one of the most common lesion sites for unilateral stroke patients that experience dysphagia [18].

The opercular cortex overlays the insula and has been reported to contribute to the swallowing process; it is one of the four cortical areas Martin et al. [8] reported that could elicit a full swallow when stimulated. The operculum contributes to the temporal organization of swallowing, such as controlling and coordinating the timing of mastication and other oral movements with swallowing [8]. This area has also been reported to be significant for sensation of the mouth and pharynx $[7,15]$.

\section{The Cerebellum and Deglutition}

The cerebellum works to monitor motor execution and effectively compare what the body intended to do with what the body actually did and make adjustments to the motor plan accordingly [14]. Axons from the SMA, PMA, MI, SI, and the posterior parietal cortex form a large projection to the pons, known as the pontine nuclei, which also relays information to the cerebellum [14]. The cerebellum modulates this information on aspects of movement direction, timing, and force and sends it back to the motor cortex by way of the thalamus [14]. Given an understanding of the established role of the cerebellum in motor coordination, timing, sequencing, and proprioceptive feedback [19], it is not unreasonable to suspect a supportive role for the cerebellum in swallowing-related functions. Conflicting data, however, have emerged in the literature on neurological swallowing disorders.

Few studies in nonhuman primate subjects have provided direct insights regarding motor movements associated with swallowing. Mussen [20] was one of the first investigators to report contributions of the cerebellum in swallowing. He reported muscle contraction at the levels of the pharynx and oral cavity evoked during the stimulation of the ventral parts of the vermis. Bernston et al. [21] much later reported wellorganized eating and grooming behavior in the cat following stimulation of regions that extended from the fastigial nucleus to the superior cerebellar peduncle. Martner [22] observed, more specifically, that stimulation of the fastigial nucleus elicited oral behaviors critical for grooming and chewing and suggested a role of the cerebellum in masticatory movements in the cat. The author further suggested that neurons of the fastigial nucleus coordinate with other regions responsible for feeding behavior. Electrical stimulation of the cerebellum has also been suggested to have inhibitory effects on cortical output related to swallowing in the cat, bolstering support for coordination between sites [23]. Gibbs [24] found less supportive results studying jaw movements and eyeblink responses in rabbits with lesioned interpositus nucleus of the cerebellum and the superior cerebellar peduncles. The researchers reported no differences in jaw movements between the experimental rabbits and sham-operated control animals. These jaw movements were, however, not purposeful masticatory movements, limiting the applicability of the findings.

Some recent studies have provided indirect inferences about the possible role of the cerebellum in sensory-motor movements associated with swallowing in nonhuman primates. For example, Strick and colleagues [25, 26] have demonstrated disynaptic connections between the basal ganglia and the cerebellum in cebus monkeys and have proposed pathways of communication between the basal ganglia and the cerebellum. The basal ganglia have been increasingly reported to have significant involvement in fine motor movements related to swallowing in primates (e.g., [27]), implying a possible contribution from the cerebellum, as well. The cerebellar connections to the cerebral cortex have been appraised, and Strick and colleagues [28] have reported widespread activation of the cerebral cortex as a result of cerebellar output, suggesting a more significant role for the cerebellum in complex motor processes such as swallowing.

The study of cerebellar functions in human subjects has largely been restricted because of the absence of monosynaptic connections between the cerebral cortex and the cerebellum, as traditional anterograde and retrograde tracing techniques cannot be effectively used to understand this relationship. Therefore, most of our understanding of the recruitment of the cerebellum in normal swallowing stems from neuroimaging paradigms and inferences from patterns of abnormalities following lesions.

\section{Cerebellum and Normal Swallowing in Human Subjects}

Research over the past decade and a half has primarily used imaging techniques such as fMRI and PET during swallowing tasks to examine neural activation patterns. In one of the earlier prospective neuroimaging studies, Zald and Pardo [1] analyzed regional cerebral blood flow (rCBF) using PET in healthy subjects during voluntary swallowing of saliva. Significant and robust activations were observed in the left inferior precentral gyrus, insula, and the left cerebellum, specifically the Crus Ia/VI region. The authors went on to compare these activations with those derived from tongue movements in another set of healthy subjects. The comparison showed 
these activations to be significantly distinct, thus, implicating the cerebellum, specifically the VI lobule, in pharyngeal swallowing.

Mosier and Bereznaya [11] proposed that swallowing occurs by activation of parallel networks and not as a hierarchically projected task. Path analyses using structural equation modeling (SEM) were performed on activations derived out of fMRI for swallowing tasks, and it was found that the cerebellum exhibits functional (and not anatomical) connectivity with the primary motor cortex, inferior frontal gyrus, basal ganglia, and thalamus for volitional swallowing. The activated structures work as independent modules, and each module performs a certain function in swallowing. Cerebellum, constituting one of the modules, may play a role in feed-forward mechanisms and controlling timing, sequencing, and internal coordination [11] for oral-lingual and pharyngeal musculature-consistent with the prior work by Zald and Pardo [1].

Suzuki et al. [12] used fMRI on 11 healthy subjects performing volitional saliva swallows in an fMRI block design. Group analysis of the images revealed bilateral cerebellar activation with predominant activation of the posterior regions of the left cerebellar hemisphere (see Fig. 1). In 2005, Harris et al. [29] observed activation of the left cerebellar hemisphere in a PET procedure assessing rCBF for volitional swallowing. Strong activations were also found in the precentral and postcentral gyri, insular gyrus, thalamus, globus pallidus, anterior cingulate gyrus, supplementary motor area, superior temporal gyrus, and substantia nigra.
Malandraki et al. [30] sought to investigate brain activations for specific tasks within a swallow. Planning for a swallow without executing it, tongue tapping, throat clearing, and a 3-ml thin liquid swallow task were employed, and neural activations were studied using fMRI. The 3-ml swallowing task induced activations in several anterior and posterior parts of the cerebellum along with several other cortical and subcortical structures. The throat-clearing task, which was believed to simulate laryngeal closure, yielded bilateral activations in the posterior parts of the cerebellum. Cerebellar involvement was not observed in the tongue tapping or planning for swallowing tasks. These findings are significant in informing us about a possible role for the cerebellum in specific tasks within swallowing.

Grabski et al. [31] also investigated the involvement of the cerebellum in supralaryngeal and laryngeal motor control. In their fMRI study, superior portions of both the cerebellar hemispheres were activated for tasks involving movement of the articulators and production of a vowel /i/. The authors proposed that comparable results were seen for articulatory movements for speech and nonspeech functions like mastication for swallowing, suggesting a positive role for the cerebellum in coordination of oral motor structures in the act of swallowing. Shibamoto et al. [32] observed activations in the cerebellum for capsule swallowing alone but not for the swallow of other bolus consistencies. The results were explained as a difference in consistency leading to different levels of motor coordination within the swallow mechanism.
Fig. 1 Cerebellar activations for healthy human swallowing demonstrated by Suzuki et al. [12]. Reproduced with permission of Springer-Verlag New York in the format journal/magazine via Copyright Clearance Center
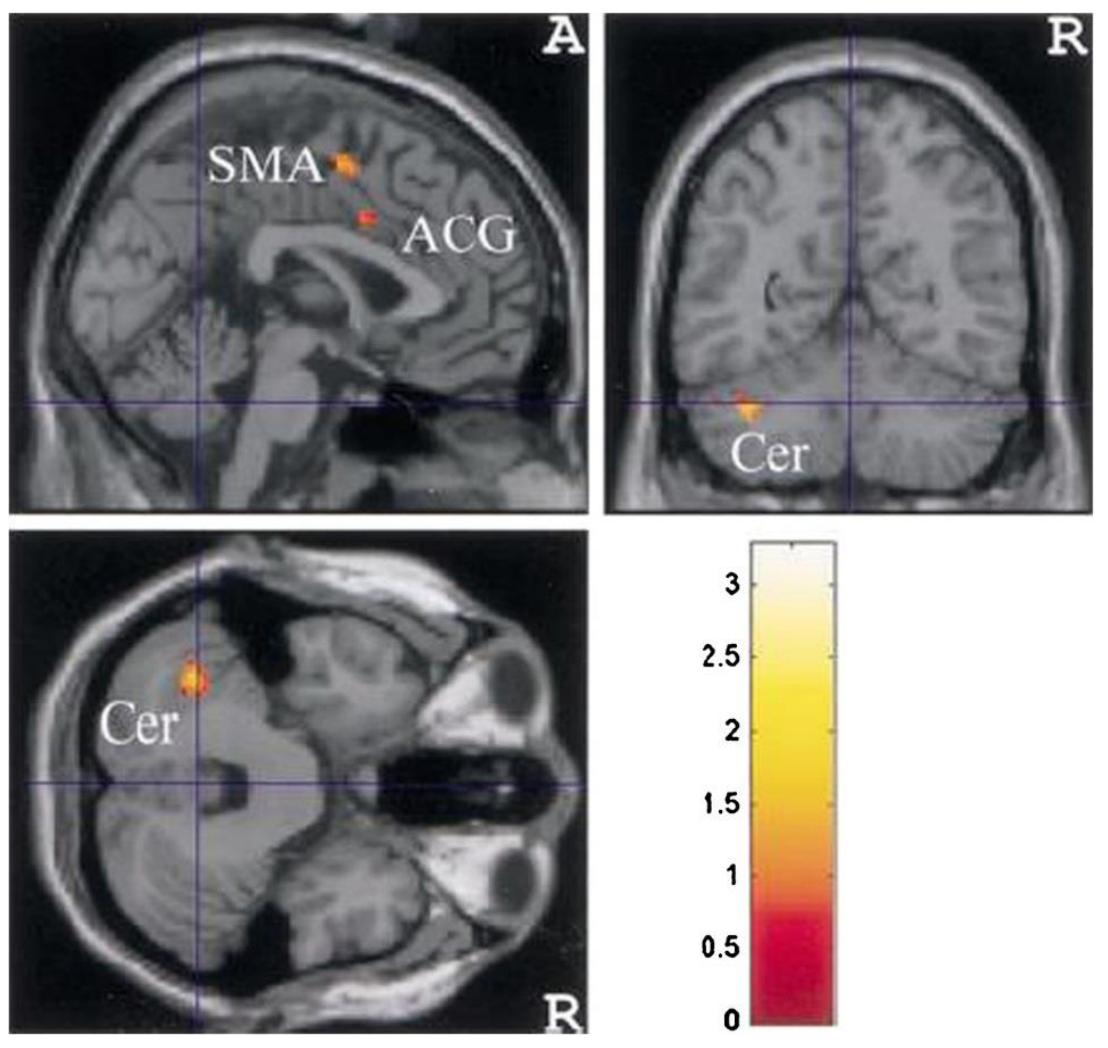
That is, it may be that the cerebellum's role in swallowing function depends on changes in task demands, which could vary from one individual to the next depending on how individuals are taxed during swallowing activities.

In a recent study, Jayasekeran et al. [33] examined the effect of TMS of the cerebellum on pharyngeal constrictor muscles. The study involved the excitation of each of the cerebellar hemispheres and the midline vermis on 16 healthy individuals. Results indicated that motor evoked potentials (MEP) were significant at the pharyngeal areas following cerebellar stimulation, although to a lesser degree than following cortical stimulation. Additionally, when cerebellar stimulation was used as a preconditioning stimulus before cortical stimulation, significant facilitation of the motor responses for cortical stimulation was observed. This implies that the cerebellum may act to intensify or modulate the cortical firing for swallowing. This may enhance our understanding of the role of TMS treatment involving the cerebellum in conjunction with cortical stimulation.

Mottolese et al. [34] attempted to map motor representation in the human cerebellum. The authors applied electrical stimulation to the posterior parts of the cerebellum to subjects undergoing surgery for a tumor outside the cerebellum. Lobule VI had strong representations of the face and mouth implying a possible representation of swallowing in the area within the cerebellum.

Most recently, Malandraki et al. (under review; personal communication) reported cerebellar activity only with sour stimuli. Some investigators believe that the cerebellum may regulate information on taste intensity with the intention to modulate oral movements during swallowing [35]. In animal studies, the cerebellum has also been reported to be involved in taste aversion learning acquisition [36]. After the fMRI portion of Malandraki et al. [30], subjects frequently reported that the sour stimulus was difficult to tolerate, and four subjects complained of reflux symptoms. The authors suggest that this was possibly reflected in the cerebellar activation seen for the stimuli that included the sour solution. Table 1 summarizes the studies demonstrating the involvement of the cerebellum in normal swallowing.

\section{The Cerebellum and Dysphagia in Human Subjects}

Few investigations report dysphagia in individuals with cerebellar lesions, and the results are largely inconclusive regarding potential patterns of dysphagia. Alberts et al. [37] attempted to correlate lesion locations with dysphagia symptoms. In their cohort of 47 subjects, 11 subjects presented with a lesion to the cerebellum-six patients with a large-vessel cerebellar lesion and five patients with small-vessel cerebellar lesions. Seven (four large-vessel lesions and three smallvessel lesions) out of these 11 patients exhibited aspiration in a swallow evaluation which was a combination of clinical bedside swallow exam and videofluoroscopic swallow exams. Min et al. [38] reported symptoms of dysphagia in only three of 31 patients with cerebellar lesions they assessed. One suffered infarctions in the proximal segment of the vertebral artery, one suffered in situ branch artery disease, and one had an area VI lesion with tandem intracranial occlusive disease. Zuketto and van Gijn [39] reported a single case study of a patient with cerebellar ectopia with severe difficulties in swallowing whose symptoms completely resolved following a compressive surgery for correction. This was consistent with studies by Massey et al. [40] and Nagahiro et al. [41] who each reported a case study of a patient with posterior cerebellar artery aneurysm presenting with dysphagia. None of these investigations reported specifics regarding type or severity of dysphagia. Perie et al. [42] reported a case study of a patient with left cerebellar hematoma and a hemorrhage of the sustentorial third ventricle. Videofluoroscopic swallow study revealed delayed swallow initiation and impaired laryngeal closure accompanied by oral and hypopharyngeal residue, as well as aspiration. In 2006, Kumral et al. [43] reported dysphagia in subjects with lesions to anterior and posterior inferior cerebellar arteries.

Recent studies, however, have provided a stark contrast. Flowers et al. [44] reported a meta-analysis of neuroanatomical predictors after acute ischemic stroke. Seventeen articles were reviewed and included for meta-analysis. A total of 656 subjects were pooled, and it was reported that the incidence of dysphagia following a lesion to the cerebellum was $0 \%$. Moon et al. [45] examined the correlation of lesion location and stages of swallow impairment in 76 patients with a stroke to different areas of the brain. Only a lesion to the medullary regions correlated with disturbances in the pharyngeal stages of swallow, especially aspiration. Vallecular residue had a moderate correlation with parietotemporal strokes. No significant dysphagia symptoms correlated with cerebellar lesions. However, it is important to note that only two subjects in this group of 76 subjects had an isolated cerebellar lesion. With such a small number, significant correlations are unlikely.

Isono et al. [46] investigated differences in dysphagia patterns in individuals with spinocerebellar ataxia type 3 (SCA3) and type 6 (SCA6). While SCA3 affects multiple systems such as the cerebellum, pyramidal, extrapyramidal, and autonomic systems, SCA6 predominantly affects the cerebellum. The authors retrospectively studied videofluoroscopic swallow exams in seven patients with SCA3 and 13 patients with SCA6. Severity of dysphagia was rated based on the dysphagia outcome severity scale (DOSS) and a regionally used scale developed by the Japanese Society of Dysphagia Rehabilitation. Results indicated that dysphagia severity, as assessed by the Japanese scale, was mild but significant in SCA6 and severe in SCA3. Moreover, DOSS outcomes indicated physiological abnormalities in SCA3 but not in SCA6. These results suggest that the dysphagia in individuals with predominant lesions to 
Table 1 Summary of studies of involvement of cerebellum in normal swallowing

\begin{tabular}{|c|c|c|}
\hline Region within the cerebellum & Function/results & Reference \\
\hline \multirow[t]{2}{*}{ Crus Ia/VI lobule region of the left hemisphere } & Volitional swallowing of saliva-pharyngeal stages & Zald and Pardo [1] \\
\hline & $\begin{array}{l}\text { Cerebellum functions in a parallel network to cortical } \\
\text { processing for volitional swallowing }\end{array}$ & Mosier and Bereznaya [11] \\
\hline $\begin{array}{l}\text { Bilateral activation, predominant in } \\
\text { posterior left hemisphere }\end{array}$ & Volitional swallowing of saliva & Suzuki et al. [12] \\
\hline Unknown & Increasing task demands recruits the cerebellum & Shibamoto et al. [32] \\
\hline Anterior and posterior parts of the cerebellum & Swallow of 3-ml liquid & Malandraki et al. [30] \\
\hline $\begin{array}{l}\text { Bilateral activations in the posterior } \\
\text { parts of the cerebellum }\end{array}$ & Throat clearing (simulation of laryngeal closure) & Malandraki et al. [30] \\
\hline $\begin{array}{l}\text { TMS of each of the cerebellar hemispheres } \\
\text { and the midline vermis }\end{array}$ & $\begin{array}{l}\text { Pharyngeal motor evoked potentials after cerebellar stimulation } \\
\text { and facilitation of the motor responses for cortical stimulation }\end{array}$ & Jayasekeran et al. [33] \\
\hline Superior portions of both the cerebellar hemispheres & Supralaryngeal and laryngeal motor control & Grabski et al. [31] \\
\hline Lobule VI & $\begin{array}{l}\text { Strong representations of the face and mouth following } \\
\text { electrical stimulation }\end{array}$ & Mottolese et al. [34] \\
\hline Both the cerebellar hemispheres & Sour stimuli only & $\begin{array}{l}\text { Malandraki et al. } \\
\text { (under review; personal } \\
\text { communication) }\end{array}$ \\
\hline
\end{tabular}

TMS transcranial magnetic stimulation

the cerebellum is not as severe as that seen with widespread lesions but does exist. Again, results may differ individually depending on consistencies employed and individual variability in response to task demands.

A few studies have looked at symptoms of dysphagia in individuals with a cerebellopontine (CP) angle tumor (e.g., $[47,48])$ and in patients following a resection of a CP angle tumor (e.g., [49]). This, however, does not inform us about the specific contribution of the cerebellum, as the tumor sites involve areas outside the actual bulk of the cerebellar tissue. Table 2 summarizes the results of studies of breakdown in swallowing following cerebellar lesions.

\section{The Cerebellum and Dysphagia Treatment}

Few recent studies address the possible role of the cerebellum in intervention for dysphagia. Ogura et al. [50] reported activation of the cerebellum in healthy subjects during the performance of oral exercises used for dysphagia in a functional magnetic resonance imaging paradigm. The study indicated significant right cerebellar activation for tongue protrusion and bilateral cerebellar involvement for lip pursing and stretching, tongue lateralization exercises, and an oral ball rolling task involving repeated right and left movements of a spherical object. The activation in the cerebellar areas occurred in conjunction with other cortical and subcortical regions, primarily the precentral and postcentral gyri and the basal ganglia. These results imply possible utility of the cerebellum in oral motor exercises used for dysphagia. However, since the participants were healthy subjects, the role of the cerebellum in the exercises for patients with dysphagia, as well as the utility of the exercises themselves, remains in question. In addition, since other sensory-motor areas were also recruited, the specific function of the cerebellum and the significance of its contribution in this cluster of structures are not clear.

Table 2 Summary of studies of breakdown in swallowing following cerebellar lesions

\begin{tabular}{|c|c|c|}
\hline Region within the cerebellum, impaired & Function/results & Reference \\
\hline Unknown & Seven out 11 patients exhibited aspiration & Alberts et al. [37] \\
\hline $\begin{array}{l}\text { Proximal segment of the vertebral artery, in situ branch artery } \\
\text { disease and area VI lesion with tandem intracranial occlusive disease }\end{array}$ & $\begin{array}{l}\text { Three out of } 31 \text { patients presented with symptoms } \\
\text { of dysphagia }\end{array}$ & Min et al. [38] \\
\hline \multirow[t]{4}{*}{ Anterior and posterior portions of the cerebellar arteries } & Symptoms of dysphagia & Kumral et al. [43] \\
\hline & Incidence of dysphagia following cerebellar lesion is $0 \%$ & Flowers et al. [44] \\
\hline & No correlation between cerebellar lesion and dysphagia & Moon et al. [45] \\
\hline & $\begin{array}{l}\text { Significantly more physiological abnormalities in } \\
\text { spinocerebellar ataxia type } 3 \text { compared to type } 6\end{array}$ & Isono et al. [46] \\
\hline
\end{tabular}


Table 3 Summary of studies of cerebellar contribution in dysphagia intervention

\begin{tabular}{lll}
\hline Region within the cerebellum & Function/results & Reference \\
\hline $\begin{array}{l}\text { Right cerebellar hemisphere for task 1 and bilateral } \\
\text { activation for tasks 2, 3, and 4 }\end{array}$ & $\begin{array}{l}\text { Task 1: Tongue protrusion } \\
\text { Task 2, 3, and 4: Lip pursing and stretching, tongue } \\
\text { lateralization exercises, and oral ball rolling }\end{array}$ & Ogura et al. [50] \\
Contralateral excitability on rTMS & $\begin{array}{l}\text { Robust excitability at 10 Hz of rTMS which also produced } \\
\text { long-lasting effects in the contralateral pharyngeal cortex }\end{array}$ & Vasant et al. [51] \\
\hline
\end{tabular}

rTMS repetitive transcranial magnetic stimulation

In a recent study, Vasant et al. [51] reported pharyngeal motor excitability following high frequency repetitive transcranial magnetic stimulation (rTMS) of the cerebellar hemispheres. Seventeen healthy adults underwent pharyngeal motor mapping with rTMS applied to the cerebellar hemispheres using neuronavigation. Bihemispheric pharyngeal cortical excitability (ipsilateral and contralateral cortex to the cerebellum site) was measured at baseline and for up to $1 \mathrm{~h}$ post cerebellar rTMS intervention at different TMS frequencies as well as during a sham stimulus. The authors reported robust excitability at $10 \mathrm{~Hz}$ of rTMS, which also produced long-lasting effects-primarily in the contralateral pharyngeal cortex. This finding suggests that $10 \mathrm{~Hz}$ rTMS may play a vital role in the intervention of neurogenic dysphagia. These authors are further investigating the utility of cerebellar rTMS in dysphagia treatment. These results provide promising data to suggest a role for the cerebellum in treatment of dysphagia even when cerebellar involvement may not be the primary cause of dysphagia. Additional research will be necessary, but stimulation of the cerebellum may be used in future patients to improve pharyngeal coordination regardless of neurogenic origin. With new technologies literally mapping out the future, such possibilities are exciting.

Table 3 includes a summary of studies demonstrating the involvement of the cerebellum in dysphagia treatment.

\section{Summary and Conclusions}

The phylogenetic origin of the human cerebellum is reported to have evolved in two ways, (a) by increasing the population of nervous cells and dendritic processes and (b) by an increase

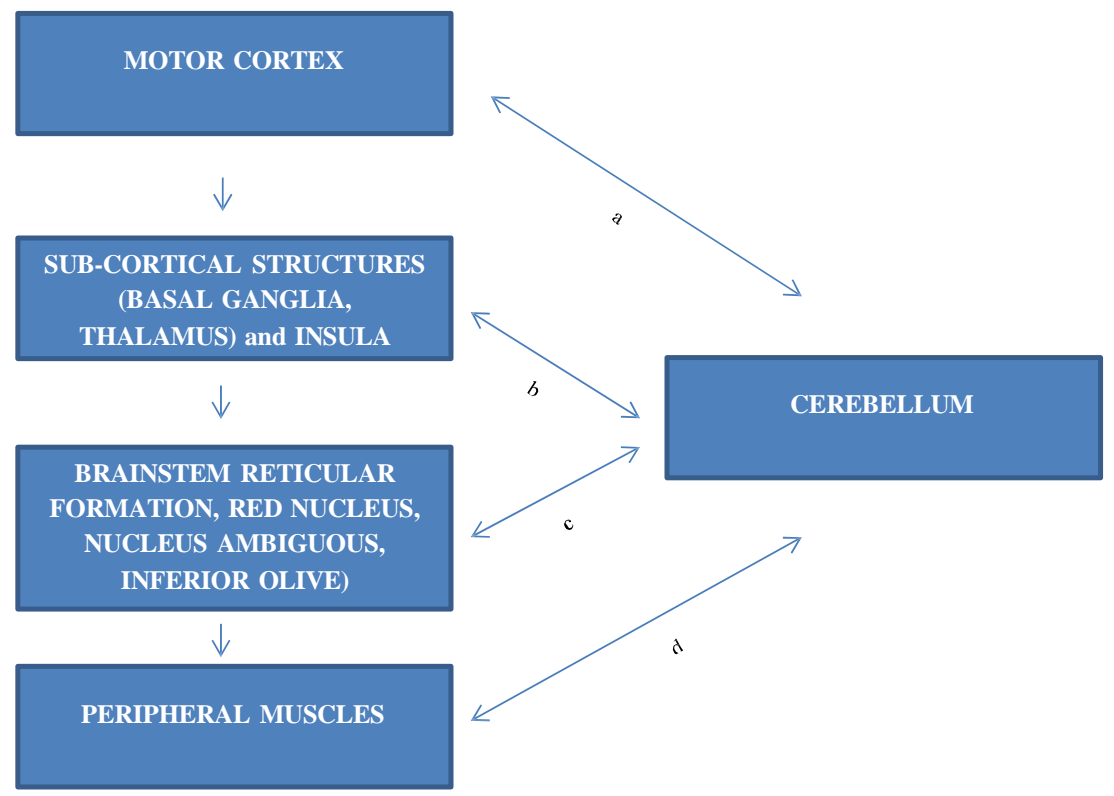

Fig. 2 Potential role for cerebellum in motor aspects of swallowing. Reciprocal motor connections of the cerebellum with other cortical, subcortical, brain stem, and peripheral structures. $a$ indicates possible contribution in coordination of oral and pharyngeal physiological movements in increased task demands like change in volume, taste, and viscosity. $b$ indicates fine motor coordination and temporal coordination of pharyngeal motor movements through possible extrapyramidal pathways. Possible direct connections to the thalamus and indirect connections to the basal ganglia, insula, for timely initiation of swallowing. $c$ indicates temporal aspects of oral and pharyngeal movements, coordination of motor output from nucleus ambiguus and inferior olivary nucleus for oral/pharyngeal motor movements. $d$ indicates feed-forward control of oral and pharyngeal movements and partial control of feedback through mossy and climbing fibers 


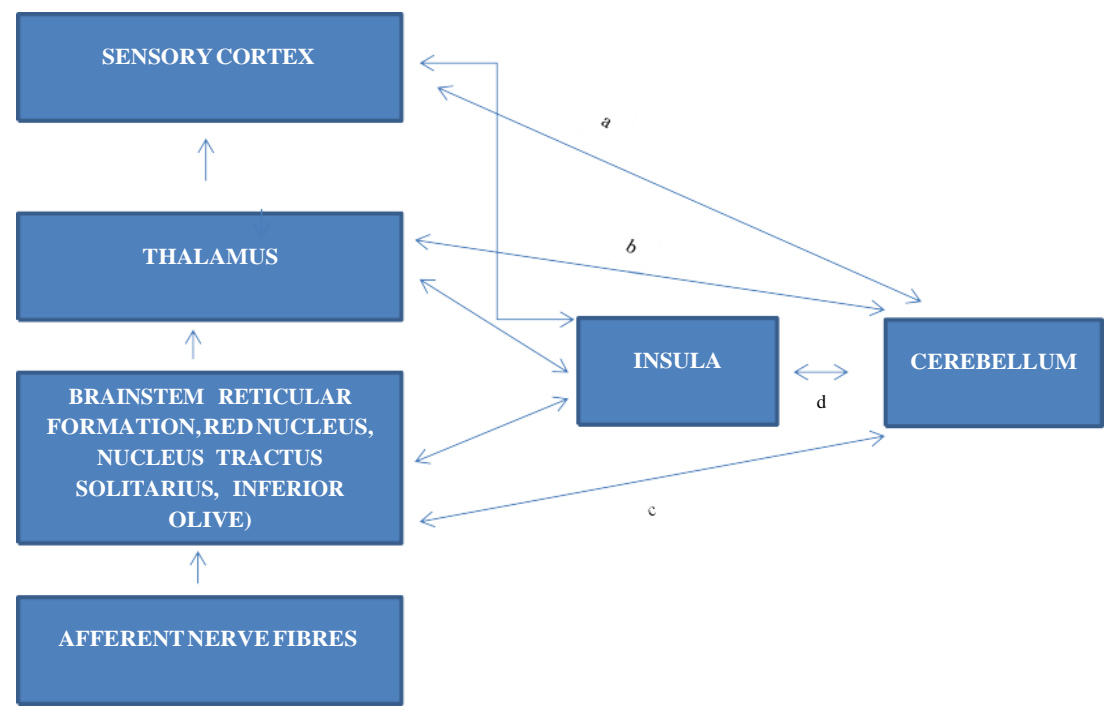

Fig. 3 Potential role for the cerebellum in sensory aspects of swallowing. Reciprocal sensory connections of the cerebellum with other cortical, subcortical, brain stem, and peripheral structures. Possible sensory augmentation of proprioceptive and gustatory senses. It is proposed that the cerebellum's connection with the cortical is predominantly descending controlling motor output with some sensory coordination. $a$ indicates

in the connections that link the cerebellum to the prefrontal cortex, thus suggesting that, for information processing, the cerebellum acts in adjunct to the cerebral cortex [52]. Figures 2 and 3 provide a schematic of the potential role of the cerebellum in motor and sensory aspects of swallowing. Studies (e.g., [53]) have suggested that the cerebellum acts as an intensifier of neural responses, coordinating selective attention, thereby aiding in the execution of commands generated in the cortex to modulate cortical output, which could be in the form of excitation or inhibition. These tenets do point to the fact that the cerebellum may compliment cortical and brainstem control for swallowing, because of the rich anatomical and functional connectivity (see Fig. 2a, c). The cerebellum has been established as a seat of feed-forward mechanisms providing internal representations to motor movements before their execution [54] (see Fig. 2d). It would be logical to expect an internal coordination of oral and pharyngeal movements, and the results of Mosier and Bereznaya [11] and Malandraki et al. [30] support this. In summary, the cerebellum appears to coordinate the cortical and brainstem output and participate in feed-forward and feedback control. Specific areas of the cerebellum appear to be involved in swallowing (see Table 1), but verification via more neuroimaging studies is necessary.

The idea of differential involvement of the cerebellum with differences in task demands cannot be excluded. Studies to address activation of the cerebellum with swallowing of different consistencies and tastes will offer further insight (see Fig. 3a, b). These neuroimaging experiments should also focus on the region of interest (ROI) analyses to understand connections to the thalamus coordinating temporal aspects and timeliness of pharyngeal swallowing. $b$ indicates reciprocal connections with the brainstem nuclei coordinating the sensation of viscosity, volume, and temporal parameters of swallowing in the pharyngeal stage. $c$ indicates possible connection to the insula contributing to initiation of pharyngeal swallowing

the interaction between cerebellar activation and the demands induced by the swallowing task. Structural equation modeling should be employed to understand covariance of activation in the cerebellum and cortical structures, which will offer insights about the functional connectivity of the cerebellum with the other structures.

Clearly, the patterns of dysphagia in patients with cerebellar lesions need to be examined more closely. Though it does appear that a lesion to the cerebellum could cause dysphagia, wide discrepancies exist within the literature. Investigations should assess specifics of temporal measures rather than assessing penetration/aspiration alone, especially given the role of the cerebellum in temporal processing and pacing [55] (see Fig. 3c, d). It would also be interesting to compare these measures with those of patients with cortical lesions. In addition, the recruitment of the cerebellum in the recovery stages is poorly understood. Studies should assess dynamics of neural activations during different stages of recovery from dysphagia following strokes to cortical centers to inform us if the cerebellum plays a compensatory role and takes part during instances of increased demands.

With ever-evolving neuroimaging and neurobehavioral techniques, our understanding of specific roles of the cerebellum in the highly coordinated and goal-oriented act of swallowing, and, more importantly, swallowing impairment, should continue to be explored and defined.

Conflict of Interest The authors declare no conflict of interest in this submission. 
References

1. Zald DH, Pardo JV. The functional neuroanatomy of voluntary swallowing. Ann Neurol. 1999;46(3):281-6.

2. Robbins J, Coyle J, Rosenbek J, Roecker E, Wood J. Differentiation of normal and abnormal airway protection during swallowing using the penetration-aspiration scale. Dysphagia. 1999;14(4):228-32.

3. Hamdy S, Mikulis DJ, Crawley A, Xue S, Lau H, Henry S, et al. Cortical activation during human volitional swallowing: an eventrelated fMRI study. Am J Physiol Gastrointest Liver Physiol. 1999;277(1):G219-25.

4. Hamdy S, Rothwell JC, Brooks DJ, Bailey D, Aziz Q, Thompson DG. Identification of the cerebral loci processing human swallowing with H2 15O PET activation. J Neurophysiol. 1999;81(4):1917-26.

5. Kern MK, Jaradeh S, Arndorfer RC, Shaker R. Cerebral cortical representation of reflexive and volitional swallowing in humans. Am J Physiol Gastrointest Liver Physiol. 2001;280(3):G354-60.

6. Kern M, Birn R, Jaradeh S, Jesmanowicz A, Cox R, Hyde J, et al. Swallow-related cerebral cortical activity maps are not specific to deglutition. Am J Physiol Gastrointest Liver Physiol. 2001;280(4): G531-8.

7. Martin RE, Goodyear BG, Gati JS, Menon RS. Cerebral cortical representation of automatic and volitional swallowing in humans. J Neurophysiol. 2001;85(2):938-50.

8. Martin RE, MacIntosh BJ, Smith RC, Barr AM, Stevens TK, Gati JS, et al. Cerebral areas processing swallowing and tongue movement are overlapping but distinct: a functional magnetic resonance imaging study. J Neurophysiol. 2004;92(4):2428-93.

9. Mosier KM, Liu W-C, Maldjian JA, Shah R, Modi B. Lateralization of cortical function in swallowing: a functional MR imaging study. Am J Neuroradiol. 1999;20(8):1520-6.

10. Mosier K, Patel R, Liu WC, Kalnin A, Maldjian J, Baredes S. Cortical representation of swallowing in normal adults: functional implications. Laryngoscope. 1999;109(9):1417-23.

11. Mosier K, Bereznaya I. Parallel cortical networks for volitional control of swallowing in humans. Exp Brain Res. 2001;140(3): 280-9.

12. Suzuki M, Asada Y, Ito J, Hayashi K, Inoue H, Kitano H. Activation of cerebellum and basal ganglia on volitional swallowing detected by functional magnetic resonance imaging. Dysphagia. 2003;18(2):71-7.

13. Toogood JA, Barr AM, Stevens TK, Gati JS, Menon RS, Martin RE. Discrete functional contributions of cerebral cortical foci in voluntary swallowing: a functional magnetic resonance imaging (fMRI) "Go, No-Go" study. Exp Brain Res. 2005;161(1):81-90.

14. Bear MF, Connors BW, Paradiso MA. Neuroscience: exploring the brain. 2001;422-32.

15. Ertekin C, Aydogdu I. Neurophysiology of swallowing. Clin Neurophysiol. 2003;114(12):2226-44.

16. Satow T, Ikeda A, Yamamoto J-I, Begum T, Thuy DHD, Matsuhashi $\mathrm{M}$, et al. Role of primary sensorimotor cortex and supplementary motor area in volitional swallowing: a movement-related cortical potential study. Am J Physiol Gastrointest Liver Physiol. 2004;287(2):G459-70.

17. Jean A. Brain stem control of swallowing: neuronal network and cellular mechanisms. Psychological Reviews. 2001;81(2):929-69.

18. Daniels SK, Foundas AL. The role of the insular cortex in dysphagia. Dysphagia. 1997;12(3):146-56.

19. Houk J, Bastianen C, Fansler D, Fishbach A, Fraser D, Reber P, et al. Action selection and refinement in subcortical loops through basal ganglia and cerebellum. Philos Trans R Soc B Biol Sci. 2007;362(1485):1573-83.

20. Mussen AT. The cerebellum. A new classification of the lobes based on their reactions to stimulation. Arch Neurol Psychiatr. 1930;23:411-61.

21. Bernston GG, Potolicchto S, Miller NE. Evidence for higher functions of the cerebellum: eating and grooming elicited by cerebellar stimulation in cats. Proc Natl Acad Sci U S A. 1973;70:2497-9.
22. Martner J. Cerebellar influences on autonomic mechanisms. An experimental study in the cat with special reference to the fastigial nucleus. Acta Physiol Scand. 1975;Suppl 425:1-42.

23. Weerasuriya A, Bieger D, Hockman $\mathrm{CH}$. Basal forebrain facilitation of reflex swallowing in the cat. Brain Res. 1979;174(1): 119-33.

24. Gibbs CM. Divergent effects of deep cerebellar lesions on two different conditioned somatomotor responses in rabbits. Brain Res. 1992;585(1):395-9.

25. Bostan AC, Dum RP, Strick PL. The basal ganglia communicate with the cerebellum. Proc Natl Acad Sci U S A. 2010;107(18):8452-6.

26. Bostan AC, Strick PL. The cerebellum and the basal ganglia are interconnected. Neuropyschol Rev. 2010;20:261-70.

27. Grillner S, Robertson B, Stephenson-Jones M. The evolutionary origin of the vertebrae basal ganglia and its role in action selection. J Physiol. 2013;591(22):5425-31.

28. Bostan AC, Dum RP, Strick PL. Cerebellar networks with the cerebral cortex and basal ganglia. Trends Cogn Sci. 2013;17(5):241-54.

29. Harris ML, Julyan P, Kulkarni B, Gow D, Hobson A, Hastings D, Zweit J, Hamdy S. Mapping metabolic brain activation during human volitional swallowing: a positron emission tomography study using [18F]fluorodeoxyglucose. Journal of Cerebral Blood Flow \& Metabolism. 2005;25(4):520-26.

30. Malandraki GA, Sutton BP, Perlman AL, Karampinos DC, Conway C. Neural activation of swallowing and swallowing-related tasks in healthy young adults: an attempt to separate the components of deglutition. Hum Brain Mapp. 2009;30(10):3209-26.

31. Grabski K, Lamalle L, Vilain C, Schwartz JL, Vallée N, Tropres I, et al. Functional MRI assessment of orofacial articulators: neural correlates of lip, jaw, larynx, and tongue movements. Hum Brain Mapp. 2012;33(10):2306-21.

32. Shibamoto I, Tanaka T, Fujishima I, Katagiri N, Uematsu H. Cortical activation during solid bolus swallowing. J Med Dent Sci. 2007;54(1):25.

33. Jayasekeran V, Rothwell J, Hamdy S. Non-invasive magnetic stimulation of the human cerebellum facilitates cortico-bulbar projections in the swallowing motor system. Neurogastroenterol Motil. 2011;23(9):831-e341.

34. Mottolese C, Richard N, Harquel S, Szathmari S, Sirigu A, Desmurget M. Mapping motor representations in the human cerebellum. Brain. 2013;136(1):330-42.

35. Small DM, Gregory MD, Mak YE, Gitelman D, Mesulam M, Parrish T. Dissociation of neural representation of intensity and affective valuation in human gustation. Neuron. 2003;39(4):701-11.

36. Mediavilla C, Molina F, Puerto A. Bilateral lesions in the cerebellar interpositus-dentate region impair taste aversion learning in rats. Physiol Behav. 1998;65(1):25-33.

37. Alberts MJ, Horner J, Gray L, Brazer SR. Aspiration after stroke: lesion analysis by brain MRI. Dysphagia. 1992;7(3):170-3.

38. Min WK, Kim YS, Kim JY, Park SP, Suh CK. Atherothrombotic cerebellar infarction vascular lesion-MRI correlation of 31 cases. Stroke J Cereb Circ. 1999;30(11):2376-81.

39. Zuketto C, van Gijn J. Severe reversible dysphagia caused by herniation of the cerebellar ectopia. Ned Tijdschr Geneeskd. 2002;146(16):771.

40. Massey CE, El Gammal T, Brooks BS. Giant posterior inferior cerebellar artery aneurysm with dysphagia. Surg Neurol. 1984;22(5):467-71.

41. Nagahiro S, Goto S, Yoshioka S, Ushio Y. Dissecting aneurysm of the posterior inferior cerebellar artery: case report. Neurosurgery. 1993;33(4):739-41.

42. Perie S, Wajeman S, Vivant R, St Guily JL. Swallowing difficulties for cerebellar stroke may recover beyond three years. Am J Otolaryngol. 1999;20(5):314-7.

43. Kumral E, Kisabay A, Atac C. Lesion patterns and etiology of ischemia in the anterior inferior cerebellar artery territory 
involvement: a clinical-diffusion weighted-MRI study. Eur J Neurol. 2006;13(4):395-401.

44. Flowers HL, Skoretz SA, Streiner DL, Silver FL, Martino R. MRIbased neuroanatomical predictors of dysphagia after acute ischemic stroke: a systematic review and meta-analysis. Cerebrovasc Dis. 2011;32(1):1-10.

45. Moon HI, Pyun SB, Kwon HK. Correlation between location of brain lesion and cognitive function and findings of videofluoroscopic swallowing study. Ann Rehabil Med. 2012;36(3):347-55.

46. Isono C, Hirano M, Sakamoto H, Ueno S, Kusunoki S, Nakamura Y. Differences in dysphagia between spinocerebellar ataxia type 3 and type 6. Dysphagia. 2013:1-6.

47. Thapa B, Hossain A, Khair A, Barua K, Hossain A, Ahmad R, et al. A clinical review of large cerebello pontine angle tumors. Bangladesh Med Res Counc Bull. 2003;29(1):23-8.

48. Wu B, Liu W, Zhu H, Feng H, Liu J. Primary glioblastoma of the cerebellopontine angle in adults: case report. J Neurosurg. 2011;114(5):1288-93.

49. Starmer HM, Best SR, Agrawal Y, Chien WW, Hillel AT, Francis HW, et al. Prevalence, characteristics, and management of swallowing disorders following cerebellopontine angle surgery. Otolaryngol Head Neck Surg. 2012;146(3):419-25.

50. Ogura E, Matsuyama M, Goto TK, Nakamura Y, Koyano K. Brain activation during oral exercises used for dysphagia rehabilitation in healthy human subjects: a functional magnetic resonance imaging study. Dysphagia. 2012;27(3):353-60.

51. Vasant D, Mistry S, Jayasekeran V, Michou E, Hamdy S. OC-036 neuronavigated repetitive cerebellar stimulation produces longlasting activation of human cortical swallowing projections. Gut. 2013;62 Suppl 1:A16-7.

52. Leiner HC, Leiner AL, Dow RS. Does the cerebellum contribute to mental skills? Behav Neurosci. 1986;100(4):443.

53. Courchesne E, Muller R, Allen G. The cerebellum: so much more. Lett Sci. 1998;282:879-80.

54. Nowak DA, Topka H, Timmann D, Boecker H, Hermsdörfer J. The role of the cerebellum for predictive control of grasping. Cerebellum. 2007;6(1):7-17.

55. Middleton FA, Strick PL. Anatomical evidence for cerebellar and basal ganglia involvement in higher cognitive function. Science. 1994;266(5184):458-61. 